\title{
Effective Power Signal Filtering using LC Filters with Air Core Coils
}

\author{
J.A.Somolinos, R. Morales, C. Morón and A. García
}

Keywords: Power Filter, Filter Design, DC sources.

\begin{abstract}
Computer controlled power supplies are usually expensive when high performance (power, wide voltage range, low ripple factor and low response time) are required. This paper presents a simple and effective computer controlled DC system based on a commercial V/f inverter combined with a three-phase bridge rectifier and a LC power filter. Because of the widely variable frequency range of the V/f inverter when a wide range of voltage is required, amorphous or ferromagnetic core coils are avoided, and air core coils become an excellent solution to implement the filter and increase the efficiency of the power system. The proposed method for designing the LC filter is based on the full dynamic model of the filter-load set and the selection of the desired poles of the system according to the load to be coupled. The proposed topology lets obtain good time responses (setting time less than $100 \mathrm{~ms}$ ) and good ripple factor (less than 1\%) without any overshooting, even when low voltage output is required.
\end{abstract}

\section{Introduction}

Computer controlled DC power sources usually offer high performance even with widely varying passive loads. This generally involves a high economic cost that may be unsuitable when the load remains fixed. In the range of some $\mathrm{kW}$, typical DC performance are about 100-800 ms of setting time with ripple factors of about $5 \%$ peak to peak. Different command signals are used for handling these sources: analog 0-10V, 4-20mA, or different standard digital IO protocols (See [1,2] a.e.). This paper presents a simple circuit topology based on a commercial V/f inverter which generates three-phase voltage outputs with constant relation Voltage/Frequency that are rectified with a noncontrolled full bridge, being nowadays relatively low cost components for the desired powers.

LC filters are widely used in power supplies of very different topologies, diverse functions (input or output filters), very variable powers and very diverse control strategies [3-4]. The use of non PWM inverters -sine wave or multilevel (see a.e [5]) - with passive filters let us reduce the presence of undesirable high frequency harmonic with any loss of efficiency.

\section{Proposed topology}

Fig. 1 shows the proposed circuit topology based on a V/f three-phase inverter with a non controlled full bridge rectifier and a power LC filter. The system uses a primary single phase AC source which limits the maximum value of its output voltage. The proposed filter is based on an air coil and a polypropylene capacitor, both elements operating correctly over a wide range of frequencies. The use of a ferromagnetic coil has to be avoided because of the variable frequency range of the rectified signals as function of the command signal. The inner coil resistance has been also considered while the inductive load is represented as a serial association of a pure coil and a resistance. The main output magnitudes are both the voltage and current over the load. In order to compare both magnitudes, a fictitious voltage computed over the resistive part of the load is used as a scaled value of the load current. 


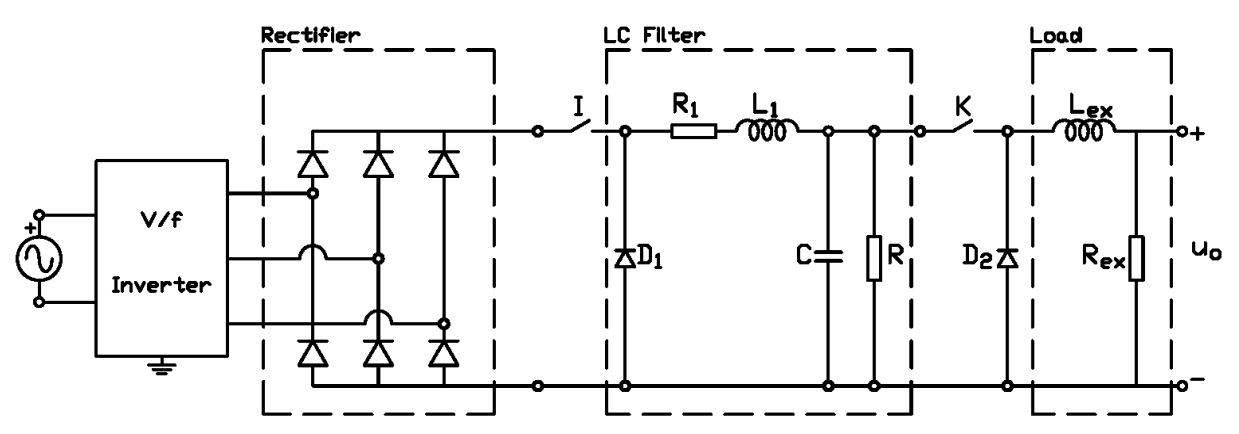

Fig. 1. Proposed Power Source System Topology with some auxiliary elements.

Two power switches (denoted as $\mathrm{I}$ and $\mathrm{K}$ ) with two anti-parallel power diodes $\left(\mathrm{D}_{1}\right.$ and $\left.\mathrm{D}_{2}\right)$ have been disposed at both sides of the filter. A power transmission line has to be inserted when the distance between the filter and the load may not be negligible. In this case, a simple serial LR impedance model was added and taken into account for computing its effects over the system.

The best position for inserting the line elements is between the common node coil-capacitor of the filter and the resistor $\mathrm{R}$ (with $\mathrm{R}>>\mathrm{R}_{\mathrm{ex}}$ ) that avoid inductive open circuits. An analog input signal over the $\mathrm{V} / \mathrm{f}$ inverter is used as the input signal of the whole system for obtaining the output voltage according to a compatible computer command signal. If a feedback controlled output is required, by measuring the desired output variable and a simple PI controller, the proposed system can be used as a feedback controlled power source with null steady state errors (See [7]).

\section{Filter design}

Amplitudes and frequencies of the 3-phase voltage outputs of the V/f inverter are given by Eq. (1)

$$
\left\{\begin{array}{l}
U_{\text {INVER }}=\sqrt{2} \cdot U_{G R I D} \cdot \frac{u_{C O M M}}{10} \cdot k_{S C A L E} \\
f_{\text {INVER }}=f_{\text {GRID }} \cdot \frac{u_{C O M M}}{10}
\end{array}\right.
$$

Where $\mathrm{u}_{\text {Сомм }}$ denotes an analog $0 . .10 \mathrm{~V}$ command signal from the DA converter of the computer, $k_{S C A L E}$ denotes the scale factor which can be selected from the V/f inverter menu for constraining the maximum voltage output, and $U_{G R I D}$ and $f_{G R I D}$ denote, respectively, the Voltage and Frequency of the mono-phase primary grid. If other linear relation between the command signal and the response of the V/f inverter is used, different relations have to be obtained. Then, if ideal diodes with a cut-in voltage $U_{\gamma}$ are considered, the time response of the rectified voltage signal is given by:

$$
u_{R E C T}(t)=\max \left\{u_{A}, u_{B}, u_{C}\right\}-\min \left\{u_{A}, u_{B}, u_{C}\right\}-2 \cdot U \gamma
$$

Where the 3-phase time responses of the V/f inverter are:

$$
\begin{gathered}
u_{A}(t)=U_{\text {INVER }} \cdot \sin \left(2 \pi f_{\text {INVER }} \cdot t\right) \\
u_{B}(t)=U_{\text {INVER }} \cdot \sin \left(2 \pi f_{\text {INVER }} \cdot t-\frac{2 \pi}{3}\right) \\
u_{C}(t)=U_{\text {INVER }} \cdot \sin \left(2 \pi f_{I N V E R} \cdot t+\frac{2 \pi}{3}\right)
\end{gathered}
$$

From Eqs. (2) and (3), the mean value of the rectified voltage is obtained as (See [6]) :

$$
U_{\text {MEAN }}=U_{\text {INVER }} \frac{6}{\pi} \sin \left(\frac{\pi}{6}\right)-2 \cdot U_{\gamma} \approx U_{\text {INVER }}
$$


On the other hand, the relation between the load voltage and its current is given by:

$u_{\text {load }}(t)=L_{\text {ex }} \cdot \frac{d i_{\text {load }}(t)}{d t}+R_{\text {ex }} \cdot i_{\text {load }}(t)$

and the output-virtual- signal is the voltage over $R_{e x}$

$u_{O}(t)=R_{e x} \cdot i_{\text {load }}(t)$

which is proportional to the load current but not directly measurable. Time response of the capacitor voltage is given by next equation.

$u_{c}(t)=u_{c}\left(t_{0}\right)+\int_{t_{0}}^{t} i_{c}(\tau) \cdot d \tau$

If Laplace transform is applied, the following relation between the signal from the rectifier and the output of the system is obtained:

$\frac{U_{0}(s)}{U_{R E C T}(s)}=\frac{1}{s^{3} a_{3}+s^{2} a_{2}+s a_{1}+a_{0}} \quad$ with $\left\{\begin{aligned} a_{3} & =\frac{L_{e x} \cdot C \cdot L_{1}}{R_{e x}} \\ a_{2} & =\left(R_{1} \cdot C \frac{L_{e x}}{R_{e x}}+L_{1} \cdot C\right) \\ a_{1} & =\left(\frac{L_{e x}}{R_{e x}}+R_{1} \cdot C+\frac{L_{1}}{R_{e x}}\right) . \\ a_{0} & =\left(1+\frac{R_{1}}{R_{e x}}\right)\end{aligned}\right.$

Under the assumption that $R_{1} \ll<R_{e x}$, Eq. (8) can be simplified and it becomes:

$\frac{U_{0}(s)}{U_{R E C T}(s)}=\frac{\frac{R_{e x}}{L_{1} C L_{e x}}}{s^{3}+s^{2}\left(\frac{R_{e x}}{L_{e x}}\right)+s\left(\frac{L_{e x}+L_{1}}{L_{1} C L_{e x}}\right)+\frac{R_{e x}}{L_{1} C L_{e x}}}$

The desired transient responses of the whole circuit (Setting time, overshooting...) are transferred into a desired denominator of the simplified Eq. (9) $\operatorname{den}(s)_{D}=s^{3}+s^{2} a_{2} D+s a_{1} D+a_{0} D$. Then, the values of the filter elements $\left(\mathrm{L}_{1}\right.$ and $\mathrm{C}$ ) are easily adjusted. It can be observed that the coefficient $a_{2} D$ is imposed by the load parameters and it is not adjustable.

\section{Time and frequency responses}

This section is devoted to show time and frequency simulated responses of the proposed system under two command signals that imply two different output voltages and frequencies from the V/f converter. The filter was tuned to $7.5 \mathrm{~Hz}$ for a $50 \mathrm{~Hz}$ grid. Smaller cutting frequencies imply components of bigger size, which is an undesirable effect.

For $u_{\text {COMM }}=1 \mathrm{~V}$, (worst case) and according with eq. (1) and (2), $U_{I N V E R}=20 \mathrm{~V}$ and $f_{I N V E R}=5 \mathrm{~Hz}$. Fig. 2 shows the ideal rectifier response and the voltage over the resistive part of the load under two conditions: i) without any kind of filter and ii) with the proposed LC filter. The frequency response of the filter-load set is drawn in Fig. 3, where the frequency responses of the system without and with the filter can be observed. The amplitude of the first harmonic $(30 \mathrm{~Hz})$ is over $-30 \mathrm{~dB}$ attenuated which is considered a good response.

Fig. 4 illustrates the voltage output of the system without and with the proposed filter when a command signal of $u_{C O M M}=5 \mathrm{~V},\left(U_{I N V E R}=100 \mathrm{~V}\right.$ and $\left.f_{I N V E R}=25 \mathrm{~Hz}\right)$ is used. It can be observed that the setting time is over $1 \mathrm{~ms}$ with a very small overshooting in both cases. 


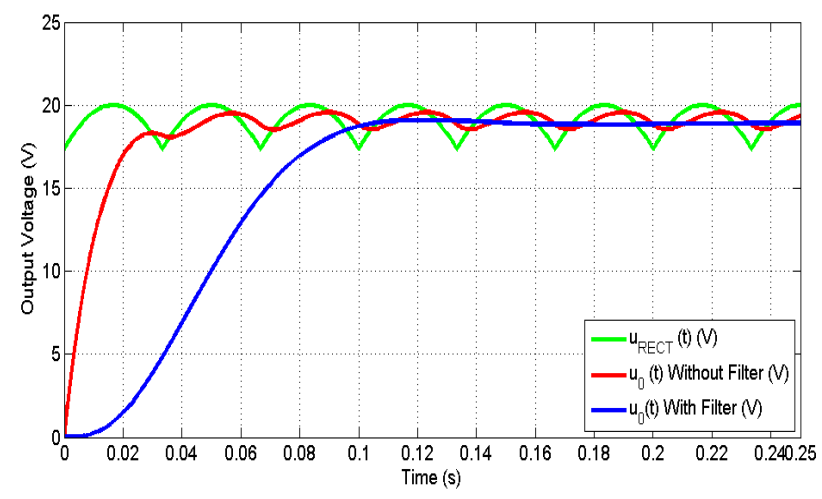

Fig. 2. Voltage time responses when $u_{\text {Сомм }}=1 \mathrm{~V}$.

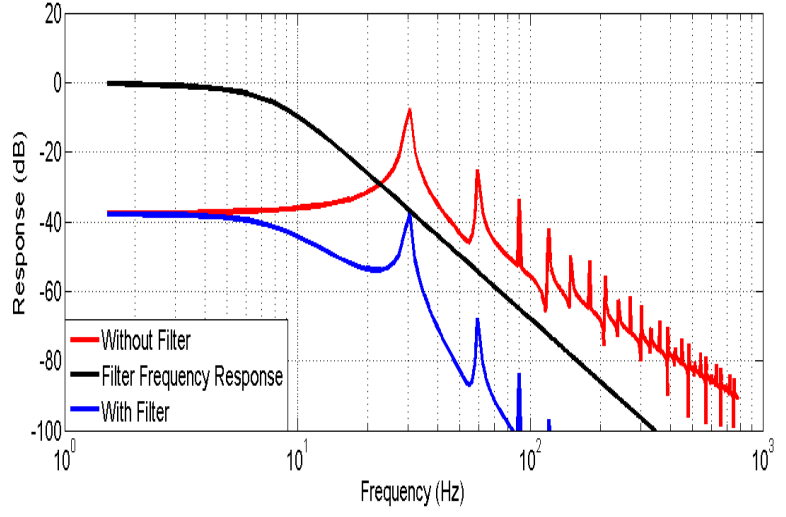

Fig. 3. Frequency responses with and without the filter when $\mathrm{u}_{\text {COMM }}=1 \mathrm{~V}$

In this second case, the first harmonic to be filtered is of $150 \mathrm{~Hz}$ and the use of the filter becomes more effective than in the first case as it can be observed in Fig. 5. By evaluating both time responses depicted in Figs. 2 and 4, it is shown that the effects of the first harmonic $(30$ and $300 \mathrm{~Hz}$ respectively) becomes unappreciable. The same conclusion could be obtained from the corresponding frequency response (attenuation over -30 and $-70 \mathrm{~dB}$ for both the first harmonics).

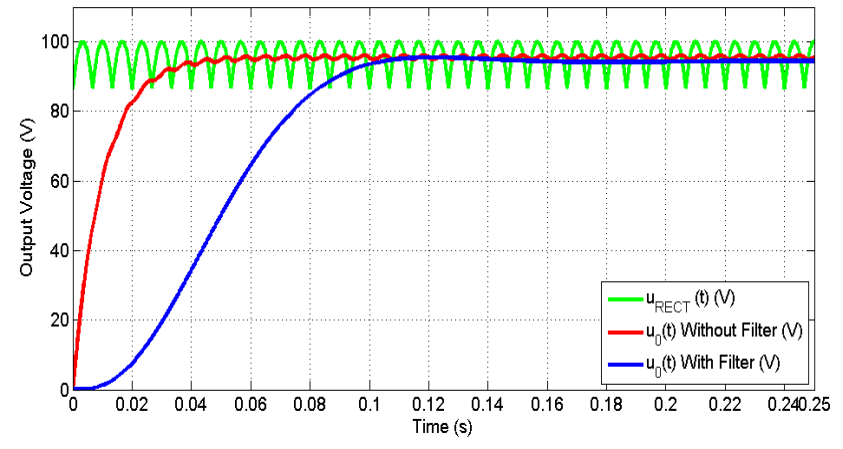

Fig. 4. Voltage time responses when $\mathrm{u}_{\text {Сомм }}=5 \mathrm{~V}$.

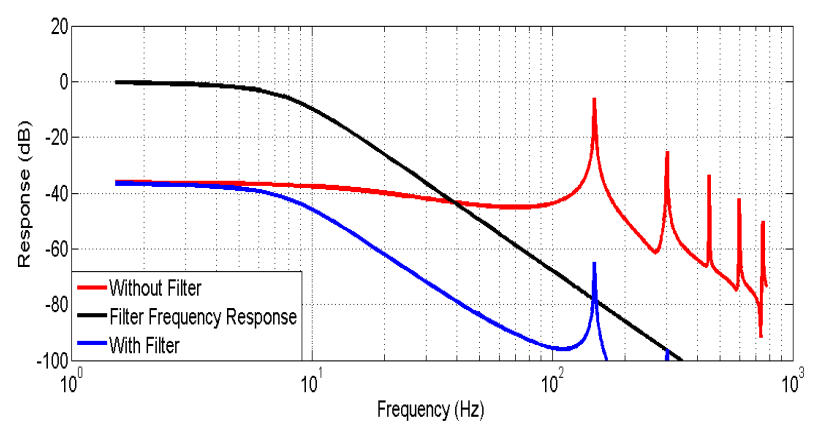

Fig.5. Frequency responses with and without the filter when $\mathrm{u}_{\text {Сомм }}=5 \mathrm{~V}$.

\section{Conclusions}

This paper studies a new circuit topology based on a V/f Inverter, a three-phase rectifier and a passive power LC filter with air core coil. A method for designing the filter as function of the load parameters and the desired response of the whole system has been presented. It is based on obtaining the linear transfer function of the full dynamics filter-load between the output of the rectifier and the load current (or load voltage) from where parameters $L_{1}$ and $C$ of the filter are obtained. Finally, simulation results under different command signal values have been illustrated implying variable amplitudes and variable harmonic frequencies.

[1] 5700/N8700 Series 1201-6600W, DC System Power Supplies. Available: on http://www.home.agilent.com.

[2] SM3300/SM6000 Series DC Power Supplies. Available: on http://www.delta-elektronika.nl.

[3] V. Blasko and V. Kaura: IEEE Trans. on Industry Applications, Vol. 33 (1997), p. 552.

[4] V. Dzhankhotov et al.: IEEE Transactions on Industrial Electronics, Vol. 58 (2011), p. 1757.

[5] J. Dixon and L. Moran IEEE Trans. on Power Electronics, Vol. 21 (2006), p. 330.

[6] N. Mohan et al.: Power Electronics: Converters, Applications, and Design. Edited by John Wiley \& Son.

[7] K. Ogata: Modern Control Engineering. $6^{\text {th }}$ Edition. Edited by Pearson. 2012 